 \\ Rundschau
}

Erstanzeige $=$ mit Raster

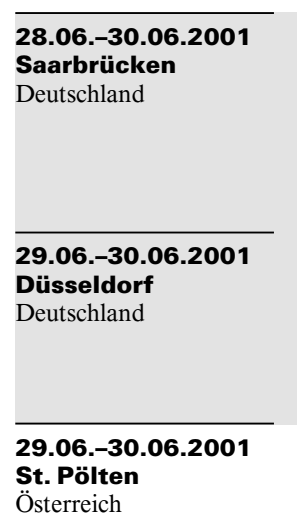

IV. Deutscher Endometriose Kongress 2001

3. Symposium

"Mammakarzinom-Behandlung - eine multidisziplinäre

Herausforderung"

V. Internationales Symposium:

Prolapsoperation in der Gynäkologie

29th British Congress of Obstetrics and Gynaecology

10.07.-13.07.200

Birmingham

Grossbritannien

\begin{tabular}{l}
\hline 28.08.2001 \\
09.10.2001 \\
27.11.2001 \\
Mainz \\
Deutschland \\
\hline 06.09.-09.09.2001 \\
Paris \\
Frankreich
\end{tabular}

\section{Ultraschallseminare 2001}

Mehrlingsschwangerschaft, Gynäkologische Sonographie, Management intrauteriner Mangelentwicklung

\section{The 2nd Congress on Controversies in Obstetrics,}

Gynecology and Infertility

07.09.-08.09.2001

Essen

Deutschland

200. Tagung der Niederrheinisch-Westfälischen

Gesellschaft für Gynäkologie und Geburtshilfe

22.09.2001

13.10.2001

08.12.2001

Solingen

Deutschland

23.09.-27.09.2001

Barcelona

Spanien

26.09.-28.09.2001

Jena

Deutschland

\subsection{9.-29.09.2001}

Nürnberg

Deutschland
Ultraschall 2001:

25. Dreiländertreffen DEGUM - SGUM - ÖGUM 5th World Congress of Perinatal Medicine
Auskunft: Ltd. OA Dr. Percy Brandner,

Dr. Christina Zwank, Frauenklinik,

Caritasklinik St. Theresia, Postfach 100534

D-66005 Saarbrücken

Tel.: +49-681-7540-281; Fax: +49-681-7540-282

E-Mail: etc.saarbruecken@t-online.de

Web: http://www.etc-gyn.de

\section{Auskunft: Frau Amelie Aengeneyndt,}

Kongressorganisation GmbH, Postfach 310307 ,

D-40482 Düsseldorf

Tel.: +49-211-47-90-439; Fax: +49-211-47-90-964

E-Mail: aa_kongress@t-online.de

Web: http://www.aa-kongress.de

Auskunft: Prim. Univ.- Doz. MR Dr. J. Lahodny,

Leiter der Geburtshilflich-Gynäkologischen Abteilung,

Allgem. Öffentl. Krankenhaus der Landeshauptstadt

St. Pölten, Propst Führer-Str. 4, Postfach 176,

A-3100 St. Pölten

Tel.: +43-2742-300-3021; Fax: +43-2742-300-3327

\section{Auskunft: C. Roney,}

Medical Conference Organisers,

Congress House, 65 West Drive,

Sutton, Surrey SM2 7NB (UK)

Tel.: +44-181-661-08-77; Fax: +44-181-661-90-36

E-Mail:info@conforg.com

Web: http://www.conforg.com

\section{Auskunft: Frau Jürgens,}

Hitachi Ultrasound GmbH, Kreuzberger Ring 21, D-65205 Wiesbaden

Tel.: +49-611-973-22-0; Fax: +49-611-973-22-10

Auskunft: Congress Secretariat,

P.O. Box 50006, Tel Aviv 61500 (Israel)

Tel.: +972-3-514-00-00; Fax: +972-3-514-00-77

E-Mail: controversies@kenes.com

Web: http://www.kenes.com/controversies/

Auskunft: Frau Amelie Aengeneyndt,

Kongressorganisation GmbH, Postfach 310307 , D-40482 Düsseldorf

Tel.: +49-211-47-90-439; Fax: +49-211-47-90-964

E-Mail: aa_kongress@t-online.de

Web: http://www.aa-kongress.de

\section{Auskunft: Frau Petra Kiefer,}

GE Ultraschall Deutschland GmbH \& Co. KG,

Postfach 110560

D-42665 Solingen

Tel.: +49-212-28-02-145; Fax: +49-212-28-02-436

Auskunft: Scientific and Social Secretariat:

Santiago Dexeus Font Foundations,

Paseo Bonanova 67, E-08017 Barcelona (Spanien)

Tel.: +34-93-227-47-09/05/00; Fax: +34-93-418-78-32

E-Mail: fundacio@iudexeus.uab.es

Web: http://www.perinatology2001.com

AGO - State of the Art, Zervix- und Endometriumkarzinom

Auskunft: Conventus Congress-Management \& Marketing GmbH, Carl-Zeiss-Platz 4,

D-07743 Jena

Tel.: +49-3641-35-88-55; Fax: +49-3641-35-88-54

E-Mail: post@conventus.de

Web: http:// www.conventus.de/ago2001

Auskunft: $\mathrm{CPO}$ Hanser Service $\mathrm{GmbH}$,

Schaumburgallee 12, D-14052 Berlin

Tel.: +49-30-30-06-69-0; Fax: +49-30-30-57-39-1

E-Mail: berlin@cpo-hanser.de

Web: http://www.cpo-hanser.de

\section{KARGER}

Fax + 41613061234 E-Mail karger@karger.ch www. karger.com
(C) 2001 S. Karger AG, Basel

Accessible online at:

www.karger.com/journals/ggr 


\subsubsection{1}

Dresden

Deutschland

28.09.-29.09.2001

Wiesbaden

Deutschland

03.10.-05.10.2001

Salzburg

Österreich

09.10 .2001

27.11.2001

Mainz

Deutschland

11.10.-13.10.2001

Wien

Österreich

\subsubsection{1}

08.12.2001

Solingen

Deutschland

19.10.-20.10.2001

Müinchen

Deutschland

11.11.2001

Mannheim

Deutschland

\subsection{1.-18.11.200}

Mainz

Deutschland

\subsection{1.-22.11.2001}

Alzey

Deutschland

23.11.-24.11.2001

\section{Wiesbaden}

Deutschland

\subsection{1.-25.11.2001}

Berlin

Deutschland

\subsubsection{1}

Mainz

Deutschland

\subsection{1.-01.12.2001}

Berlin

Deutschland
Frühdiagnostik des Zervixkarzinoms

Kolposkopie - Einführungs- und Fortgeschrittenenkurs

XVII. Akademische Tagung deutschsprechender Hochschullehrer in der Gynäkologie und Geburtshilfe

\section{Ultraschallseminare 2001}

Gynäkologische Sonographie, Management intrauteriner Mangelentwicklung

\section{Menopause 2001: Andropause}

Europas Menopausekongress

Der Fet im Blickpunkt - Ultraschall-Kolleg 2001

Hydrops fetalis, 3-D

\section{Mammasonographiekurs zur Fort- und Weiterbildung} gemäss den Richtlinien der KBV und DEGUM

Grund-, Aufbau- und Abschlusskurs Doppler-Sonographie nach den Richtlinien der KBV

Mainzer Dopplerkurse 2001: Geburtshilfe und Pränatalmedizin Abschlusskurs

\section{Urogynäkologischer Aufbaukurs}

(Urogynäkologie II - Theorie and Praktikum)

Ultraschall-Kurse Mammasonographie 2001

Abschlusskurs

\section{Mammasonographie-Kurse 2001}

Abschlusskurs

\section{Ultraschallseminare 2001}

Management intrauteriner Mangelentwicklung

20. Deutscher Kongress für perinatale Medizin
Auskunft: OÄ Dr. med. Barbara Richter,

Sekretariat Frau Brückner,

Universitäts-Frauenklinik, Fetscherstrasse 74,

D-01307 Dresden

Tel.: +49-351-458-28-64/28-05

Fax: +49-351-458-57-60

Auskunft: Dr. med. Hk. Bauer,

Biebricher Allee 35,

D-65187 Wiesbaden

Tel.: +49-611-81-18-81; Fax: +49-611-84-16-80

Auskunft: PROCON Conference,

Incentive and Event Management $\mathrm{GmbH}$,

Frau C. Satke,

Odoakergasse 34-36/3, A-1160 Wien

Tel.: +43-1-486-40-40-45; Fax: +43-1-486-40-40-46

E-Mail: office@proconference.at

Auskunft: Frau Jürgens,

Hitachi Ultrasound GmbH, Kreuzberger Ring 21,

D-65205 Wiesbaden

Tel.: +49-611-973-22-0; Fax: +49-611-973-22-10

Auskunft: Ärztezentrale Med.Info,

Helferstorferstrasse 4, A-1010 Wien

Tel.: +43-1-531-16-31; Fax: +43-1-531-16-61

E-Mail: azmedinfo@media.co.at

Auskunft: Frau Petra Kiefer,

GE Ultraschall Deutschland GmbH \& Co. KG,

Postfach 1105 60,

D-42665 Solingen

Tel.: +49-212-28-02-145; Fax: +49-212-28-02-436

Auskunft: Dr. med. Weyerstahl,

Postfach 101511 ,

D-80089 München

Tel.: +49-89-50-80-60-60; Fax: +49-89-50-80-60-77

Auskunft: Frau H. Riegler,

Sekretariat Prof. Dr. med. F. Melchert,

Klinikum Mannheim GmbH,

Theodor-Kutzer-Ufer 1-3,

D-68167 Mannheim

Tel.: +49-621-383-22-86; Fax: +49-621-383-38-14

E-Mail: heide.riegler@gyn.ma.uni-heidelberg.de

\section{Auskunft: Frau Jürgens,}

Hitachi Medical Ultrasound $\mathrm{GmbH}$,

Kreuzberger Ring 21 ,

D-65205 Wiesbaden

Tel.: +49-611-973-22-0; Fax: +49-611-973-22-10

\section{Auskunft: Dr. Rainer Lange,}

Antonitersstrasse 55,

D-55232 Alzey

Tel.: +49-6731-4-20-31; Fax: +49-6731-4-20-32

E-Mail: lange-alzey@t-online.de

Auskunft: PD Dr. med. H. Madjar,

DKD Deutsche Klinik für Diagnostik,

Aukammallee 33 ,

D-65191 Wiesbaden

\section{Auskunft: Frau Rinner,}

Schering Deutschland $\mathrm{GmbH}$,

Tel.: +49-30-349-89-243; Fax: +49-30-349-89-271

oder:

Frau Rieck, UFK Charité Berlin,

Tel.: +49-30-28-02-42-84; Fax: +49-30-28-02-84-88

Auskunft: Frau Jürgens,

Hitachi Ultrasound GmbH, Kreuzberger Ring 21,

D-65205 Wiesbaden

Tel.: +49-611-973-22-0; Fax: +49-611-973-22-10

\section{Auskunft: Frau Amelie Aengeneyndt,}

Kongressorganisation $\mathrm{GmbH}$,

Postfach 3103 07, D-40482 Düsseldorf

Tel.: +49-211-47-90-439; Fax: +49-211-47-90-964

E-Mail: aa kongress@t-online.de

Web: http://www.aa-kongress.de 
29.11.-01.12.2001

Berlin

Deutschland

30.11.-02.12.2001

Berlin

Deutschland

\section{Paris}

Frankreich

25.04.-28.04.2002

Tübingen/Berlin

Deutschland

26.04.-28.04.2002

Berlin

Deutschland

Innovative Surgery

\subsection{6.-14.06.2002}

Berlin

Deutschland

\subsection{9.-14.09.2002}

Düsseldorf

Deutschland

54. Kongress der DGGG

(Deutsche Gesellschaft für Gynäkologie und Geburtshilfe)

\section{0th World Congress on the Menopause}

omatische Geburtshilfe und Gynäkologie -

Fortbildungsveranstaltung der DGPFG Berlin

Der Fet im Blickpunkt - Ultraschall-Kolleg 2001

3-D

1res Journées Interactives de Réalités en

Gynécologie-Obstétrique (Jirgo)

World Congress of Gynecological Endoscopy and

World Congress on Gynecological Endoscopy and

Innovative Surgery

20. Jahrestagung der Deutschen Gesellschaft für Senologie
21.09.-26.09.2003 Santiago

Chile
Auskunft: CTW-Congress Organisation,

Thomas Wiese GmbH, Gosslerstrasse 30 ,

D-12161 Berlin

Tel.: +49-30-85-99-620; Fax: +49-30-85-07-98-26

Auskunft: Fr. Dr. med. M. Rauchfuss,

Frau Lilien Schelensky, Sekretariat,

Ammerseestrasse 22,

D-12527 Berlin

Tel. Fax: +49-30-624-85-52

Auskunft: Frau Petra Kiefer,

GE Ultraschall Deutschland GmbH \& Co. KG,

Postfach 1105 60,

D-42665 Solingen

Tel.: +49-212-28-02-145; Fax: +49-212-28-02-436

Auskunft: Performances Médicales,

91, avenue de la République,

F-75540 Paris cedex 11 (France)

Tel.: +33-1-47-00-67-14; Fax: +33-1-47-00-69-99

E-Mail: jirgo@performances-medicales.com

Auskunft: Congress Project Management $\mathrm{GmbH}$ Letzter Hasenpfad 61

D-60598 Frankfurt/Main

Tel.: +49-69-6090-95-31; Fax: +49-69-6090-95-40

E-Mail: cpm.sachs.ffm@t-online.de

Web: http://www.world-congress-endoscopy-2002.com

Auskunft: Congress Project Management GmbH,

Letzter Hasenpfad 61,

D-60598 Frankfurt/Main

Tel.: +49-69-60-90-95-31; Fax: +49-69-60-90-95-40

E-Mail: cpm.sachs.ffm@t-online.de

Web: http://www.world-congress-endoscopy-2002.com

Auskunft: CPO Hanser Service $\mathrm{GmbH}$,

Schaumburgallee 12, D-14052 Berlin

Tel.: +49-30-30-06-69-0; Fax: +49-30-30-57-39-1

E-Mail: berlin@cpo-hanser.de;

Web: http://www.cpo-hanser.de

\section{Auskunft: CPM Congress Project}

Management GmbH, Frau K. Sachs,

Letzter Hasenpfad 61,

D-60598 Frankfurt/Main

Tel.: +49-69-6090-95-31; Fax: +49-69-6090-95-40

E-Mail: cpm.sachs.ffm@t-online.de

Auskunft: Congress Secretariat EIMP,

759 Square Victoria, Suite 300

Montréal, Quebec, H2Y 2 J7 (Canada)

Tel.: +1-514-286-08-55; Fax: +1-514-286-60-66

E-Mail:info@eventsintl.com;

Web: http://www.eventsintl.com 